\title{
Effective Connectivity of the Human Cerebellum during Visual Attention
}

\author{
Thilo Kellermann, ${ }^{1,2}$ Christina Regenbogen, ${ }^{1,2}$ Maarten De Vos, ${ }^{3,4}$ Carolin Mößnang, ${ }^{1,2}$ Andreas Finkelmeyer, ${ }^{1,2,5}$ \\ and Ute Habel ${ }^{1,2}$ \\ ${ }^{1}$ Department of Psychiatry, Psychotherapy and Psychosomatics, Rheinisch-Westfaelische Technische Hochschule (RWTH) Aachen University, D-52074

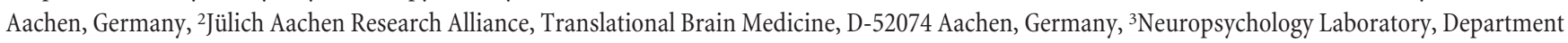 \\ of Psychology, University of Oldenburg, D-26111 Oldenburg, Germany, ${ }^{4}$ Department of Electrical Engineering, ESAT-SCD, Katholieke Universiteit Leuven, \\ 3000 Leuven, Belgium, and 5 Institute of Neuroscience, Newcastle University, Newcastle Upon Tyne NE1 7RU, United Kingdom
}

Insights from both lesion and neuroimaging studies increasingly substantiate the view that the human cerebellum not only serves motor control but also supports various cognitive processes. Higher cognitive functions like working memory or executive control have been associated with the phylogenetically younger parts of the cerebellum, crus I and crus II. Functional connectivity studies corroborate this notion as activation of the cerebellum correlates with activity in numerous areas of the cerebral cortex. Moreover, these cerebrocerebellar loops were shown to be topographically organized. We used an attention-to-motion paradigm to elaborate on the effective connectivity of cerebellar crus I during visual attention. Psychophysiological interaction analyses demonstrated enhanced connectivity of the cerebellum-during attention - with dorsal visual stream regions including posterior parietal cortex (PPC) and left secondary visual cortex (V5). Dynamic causal modeling revealed a modulation of the connections from V5 to PPC and from crus I to V5 by attention. Remarkably, the influence which V5 exerted on PPC was reduced during attention, resulting in a suppression of the sensitivity of PPC to bottom-up information. Moreover, the sensitivity of V5 populations to inputs from crus I was increased under attention. This might underscore the presumed role of the cerebellum as a state estimator that provides hierarchically lower regions (V5) with top-down predictions, which in turn might be based on endogenous inputs from PPC to the cerebellum. These results are in line with formulations of attention in predictive coding, where attention increases the precision or sensitivity of hierarchically lower neuronal populations that may encode prediction error.

\section{Introduction}

The role of the cerebellum in cognitive functions had already been raised at least 20 years ago (Schmahmann, 1991). Since then, numerous neuroimaging studies have been published that report cerebellar activation during various cognitive tasks in healthy volunteers. Cognitive domains that have been associated with the cerebellum include language (Frings et al., 2006), spatial processing (Graydon et al., 2005), working memory (Chen and Desmond, 2005), and executive functions (Blackwood et al., 2004; for review, see Stoodley and Schmahmann, 2009). In addition, investigations of the functional connectivity of the cerebellum in the resting brain yielded different cerebrocerebellar loops

\footnotetext{
Received Feb. 13, 2012; revised June 21, 2012; accepted June 23, 2012.

Author contributions: T.K. designed research; T.K., C.R., C.M., and A.F. performed research; T.K., M.D.V., C.M., A.F., and U.H. contributed unpublished reagents/analytic tools; T.K., C.R., and M.D.V. analyzed data; T.K., C.R., and U.H. wrote the paper.

This work was supported by the Deutsche Forschungsgemeinschaft (DFG, IRTG 1328, KF0-112/TP9: Ha3202/22), the Helmholtz Alliance "Mental Health in an Ageing Society" and the Interdisciplinary Center for Clinical Research of the Medical Faculty of the RWTH Aachen University (N2-6, N4-4). We are very grateful to the anonymous reviewers for improvements suggested on an earlier draft of this manuscript.

The authors declare no competing financial interests.

Correspondence should be addressed to Dr. Thilo Kellermann, Department of Psychiatry, Psychotherapy and Psychosomatics, RWTH Aachen University, Medical School, Pauwelsstrasse 30, D-52074 Aachen, Germany. E-mail: tkellermann@ukaachen.de.

DOI:10.1523/JNEUROSCI.0678-12.2012

Copyright $\odot 2012$ the authors $\quad 0270-6474 / 12 / 3211453-08 \$ 15.00 / 0$
}

which are assumed to underlie a functional topography of the cerebellum (Habas et al., 2009, Krienen and Buckner, 2009, O'Reilly et al., 2010). Of particular importance for the present study is the "executive control network," which comprises neocerebellar crus I, dorsolateral as well as dorsomedial prefrontal cortex, and superior parietal cortex (Habas et al., 2009). Recently, distinct activation of this network has been demonstrated during a working memory task, where load-dependent activity in crus I/II correlated with accordant changes in reaction times (Salmi et al., 2010).

Given this involvement of the cerebellum in such a wide variety of cognitive and sensorimotor tasks, it is quite intriguing that lesions in the cerebellum indeed lead to corresponding impairments, yet they usually do not go along with a complete loss of specific functionality (Schmahmann and Sherman, 1998). These observations led to the conclusion that the cerebellum is a "useful but not necessary" controller of sensory data acquisition (Bower, 1997). Others emphasized the importance of the temporal accuracy of ongoing interaction of the organism with a dynamically changing environment in which the cerebellum plays a critical role for an efficient and smooth execution (Ivry, 1997; Paulin, 1997). More recently, this hypothesis was corroborated by evidence of enhanced connectivity of lobule VII with the posterior parietal cortex, frontal eye fields, and dorsolateral prefrontal cortex during the timing of moving visual stimuli (O'Reilly et al., 
2008). The authors concluded that crus I supplies a temporal signal to cortical networks engaged in spatial orienting. Analyses of effective connectivity of the cerebellum are hitherto very scarce, but biological motion perception has been reported to modulate cerebellar input to superior temporal sulcus (Sokolov et al., 2012) and rhyming modulated reciprocal connections between the cerebellum and frontal as well as temporal regions (Booth et al., 2007).

The present imaging study probes the contribution of the cerebellum to visual attention during a simple attention-to-motion task (Büchel et al., 1998). A psychophysiological interaction analysis was used to identify cortical regions whose cerebellar inputs were modulated by attention. A subset of this network was then further examined using dynamic causal modeling to characterize the modulatory effects of attention. We hypothesized that cerebellar afferents to the dorsal visual stream would show attentional modulation. Specifically, we hypothesized that intrinsic processing in the cerebellum would show attention-related effects and that the output of this processing to motion-sensitive areas in the visual system would be enabled selectively during attention.

\section{Materials and Methods}

Subjects. Sixteen healthy volunteers ( 9 males, 7 females) participated in this study. All subjects were right-handed, had no history of psychiatric or neurological illness, and were aged between 22 and 32 years (mean 25.4 years, SD 2.9 years). The study was conducted in compliance with the Declaration of Helsinki and the local Institutional Review Board approved the experimental protocol. Written informed consent was obtained from every subject before participation in this study.

Stimuli and task. The baseline consisted of a white frame $\left(22.13^{\circ} \times\right.$ $\left.11.68^{\circ}\right)$ on a black background with a red fixation cross in the middle presented for $10-13.5 \mathrm{~s}$ (mean $12 \mathrm{~s}$ ) on the screen. In the STATIONARY condition nine vertically oriented white bars were presented within the white frame. One bar covered a visual angle of $\sim 0.76^{\circ} \times 11.04^{\circ}$ and the distance between two bars amounted to $1.91^{\circ}$. Participants were instructed to simply fixate the red cross. During the two other conditions, these bars moved horizontally (either from right to left or from left to right) with a speed of $\sim 5.8^{\circ}$ per second (Fig. 1 ). In one of these two conditions, subjects were instructed to fixate the red cross and refrain from ocular movement (FIX-MOVING). During the last condition, participants also saw these moving bars but were instructed to attend to slight changes in the velocity of the bars (ATTEND-MOVING). In fact, however, the speed of the bars did not change at all during the blocks inside the scanner.

During a short training session outside the scanner the subjects were familiarized with the stimuli and the task. Short instructions (2s) preceded each block indicating whether the subject had to fixate the following stimuli or whether they had to attend to changes in velocity of moving bars. Each activation condition had a length of $16 \mathrm{~s}$ and was preceded by a baseline. The STATIONARY condition was shown 9 times during the experimental run and the other two conditions (FIX-MOVING and ATTEND-MOVING) were presented 8 times each. The total length of this run (including 3 prescans) was $720 \mathrm{~s}$, where the first and the last baselines were presented for 8 and $16.5 \mathrm{~s}$, respectively.

Data acquisition. Functional magnetic resonance imaging (fMRI) was performed with a Siemens Trio MRI scanner with 3 Tesla. Functional images were acquired with a $2^{*}$-weighted echoplanar imaging (EPI) sequence consisting of 33 slices with a thickness of $3.4 \mathrm{~mm}$ (gap between slices: $0.51 \mathrm{~mm}$ ) and an in-plane resolution of $64 \times 64$ pixels (field of view: $200 \mathrm{~mm}$, voxel-size: $3.125 \times 3.125 \times 3.4 \mathrm{~mm}^{3}$ ). Orientation of the slices was parallel to the AC-PC (anterior commissureposterior commissure) line and they were assessed in ascending order. A total of 360 functional volumes were measured using the following parameters: TR: $2000 \mathrm{~ms}$, TE: $30 \mathrm{~ms}$, flip-angle: $76^{\circ}$. The first three images of each time-series were discarded due to T1 stabilization effects. Anatomical images were acquired using a T1-weighted magnetization prepared rapid gradient echo (MPRAGE) sequence pro-

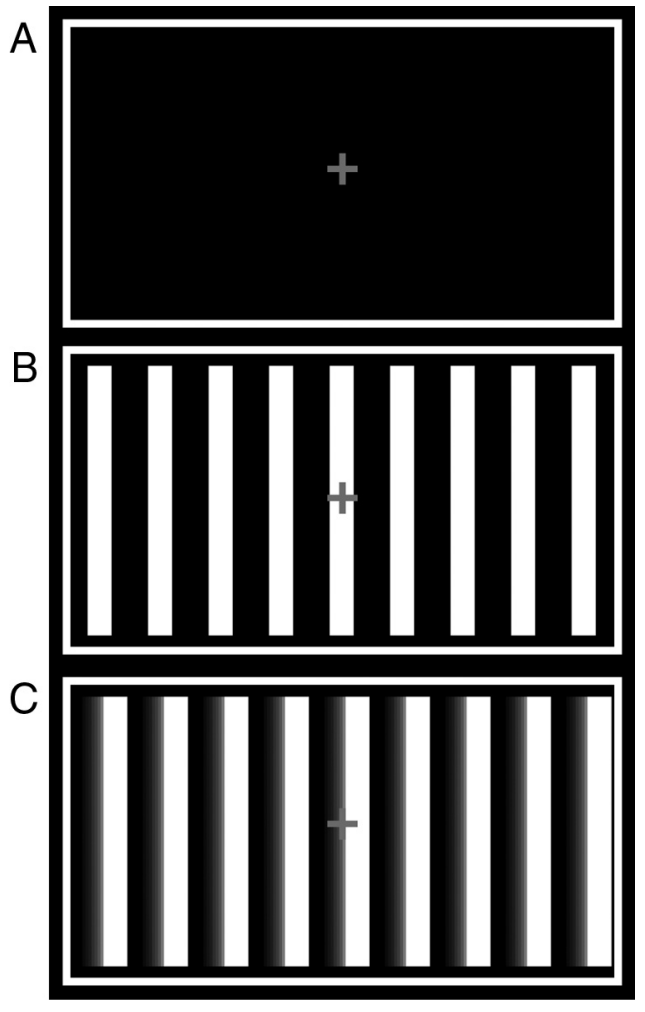

Figure 1. Illustration of the stimuli during the experiment. $A$, Depiction of the baseline condition during which only a red fixation cross in a white frame was presented. $\boldsymbol{B}$, During the STATIONARY condition, nine static bars were presented in addition to the baseline stimuli. $\boldsymbol{C}$, Stimuli of the other two conditions were identical, where the bars moved in either of two directions (left or right). During FIX-MOVING, participants were instructed to just fixate the red cross in the middle of the screen. Contrariwise, subjects had to watch out for changing velocity of the moving bars during the ATTEND-MOVING condition.

viding isotropic voxels of $1 \mathrm{~mm}^{3}$ with the following parameters: TR: $1900 \mathrm{~ms}$, TE: $2.52 \mathrm{~ms}$, flip-angle: $9^{\circ}$.

Participants were told to refrain from any movement of the limbs to avoid cerebellar activation due to motor activity. Therefore, attentionrelated changes in the alpha frequency band were assessed by means of electroencephalography (EEG) which was recorded simultaneously during fMRI scanning. As expected, a desynchronization in the individual alpha band was observed in the comparison of the FIX-MOVING and the ATTEND-MOVING conditions. This result constitutes a physiological validation of our attentional manipulation.

Data preprocessing and general linear model analysis. Preprocessing and analysis of the MR images was performed with SPM8 (Wellcome Trust Centre for Neuroimaging, London) implemented in Matlab 7.7 (The MathWorks). The images of the time-series were realigned with a twopass procedure, where the first image (first pass) and the mean image (second pass) were used as reference. Each anatomical scan was coregistered to its according mean EPI scan, which was afterward used to determine spatial normalization parameters by means of the unified segmentation approach (Ashburner and Friston, 2005). These normalization parameters were applied to the functional scans and thus transformed the time-series into the standard space defined by the Montreal Neurological Institute (MNI). Spatial normalization of the cerebellum was accomplished using the SUIT toolbox (Diedrichsen, 2006). During normalization all images were resampled to a voxel size of $1.5 \times 1.5 \times 1.5$ $\mathrm{mm}^{3}$ and afterward, images were smoothed with an isotropic Gaussian kernel of $8 \mathrm{~mm}$ full width at half-maximum.

Individual time-series were analyzed (first level) within the framework of the general linear model (GLM). Four box car functions (one for each of the three conditions and one for the preceding instructions) were convolved with the canonical hemodynamic response function (HRF) and then used as predictors in the GLM. The six 


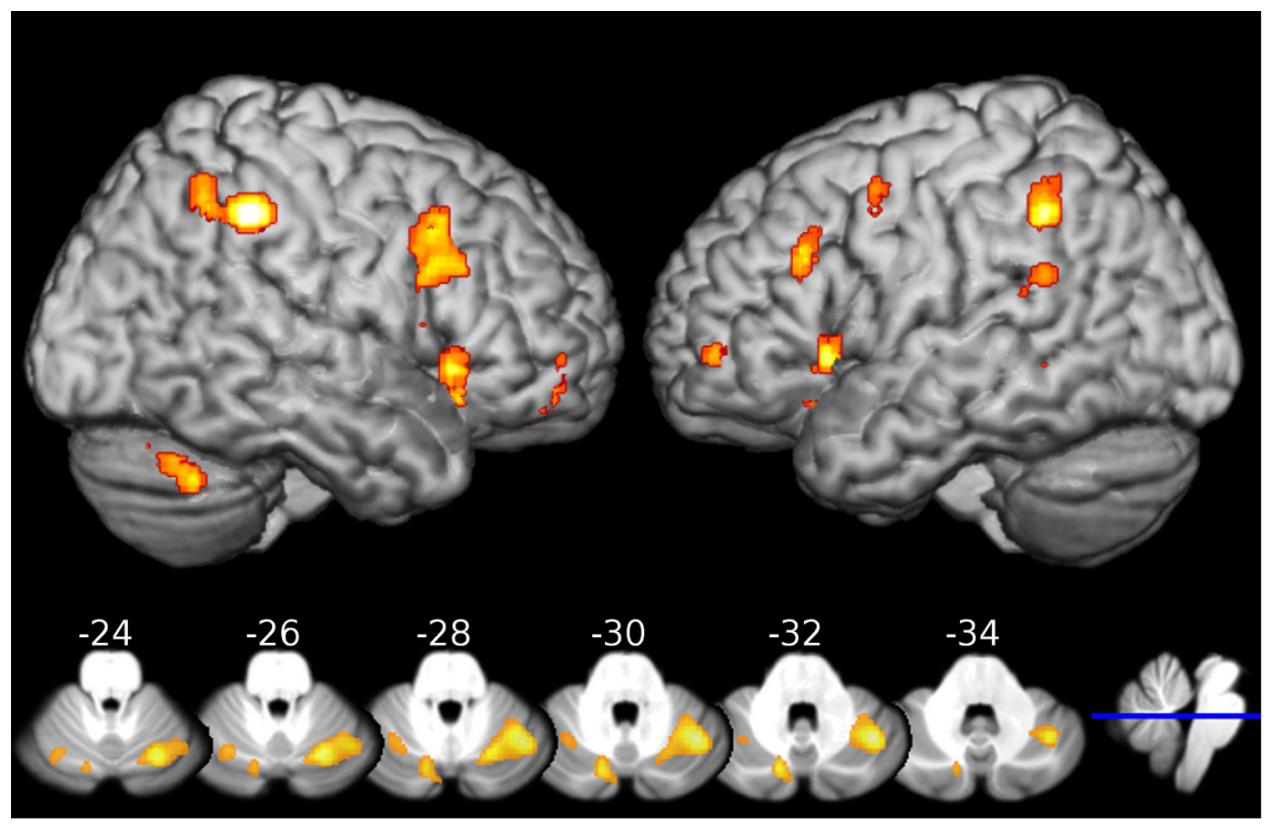

Figure 2. Activation clusters related to attention to moving objects. The top shows the exterior cortical activations rendered on an anatomical brain image (view from right and from left, respectively). One large activation cluster in the medial prefrontal cortex is not visible (see Table 1 for details). The bottom depicts selected axial slices of the same activation within the cerebellum overlaid on the unbiased template from the SUIT toolbox (Diedrichsen, 2006). The numbers above each slice indicate the level of the section plane in MNI space. The blue line on the sagittal slice on the right indicates the level at $z=-30 \mathrm{~mm}$. Analyses for the whole brain and for the cerebellum were both thresholded at $p<0.05$ corrected at the voxel level. The larger activations for the analysis restricted to the cerebellum are for the most part due to the reduced search volume.

realignment parameters (translations and rotations along the three spatial dimensions) of each scan were used as covariates to remove residual artifacts due to head movements. The mean across time in each voxel was modeled by a constant term and low-frequency drifts were removed using a high-pass filter with a cutoff period of $128 \mathrm{~s}$. Temporal correlations were modeled by a first order regression process in the usual way.

The resulting images containing the parameter estimates of the three conditions of each subject were entered into a repeatedmeasures ANOVA with mixed effects. The factor "subject" was used for random effects and the three conditions served as levels of the fixed effects factor. The contrast "attend-moving-bars" versus "fixate-moving-bars" (ATTEND-MOVING vs FIX-MOVING) was thresholded at $p<0.05$ (familywise error corrected).

To establish the attentional modulations of connections within an extended cerebellar-dorsal cortical network, we pursued the following strategy. First, using the conventional whole-brain SPM analysis described above, we identified cerebellar regions showing attentiondependent responses. We then used a psychophysiological interaction (PPI) analysis to identify cortical regions whose (polysynaptic) cerebellar inputs were modulated by attention. To characterize the modulatory effects of attention among a subnetwork of the dorsal visual stream (see Results) and the cerebellum, we used dynamic causal modeling (DCM) within this extended system.

Psychophysiological interaction between cerebellar time-course and task. The PPI analyses were used to identify cortical regions for subsequent modeling that showed psychological (attentional) effects on linear estimates of coupling -in terms of attention-dependent changes in the influence of remote (cerebellar) influences. Therefore, we extracted the high-pass filtered and adjusted time course (effects from the intercept and realignment parameters were removed) from the cerebellum of each individual time-series. These vectors were deconvolved and multiplied with the box car function representing the difference between attend- and fixate-moving-bars (ATTENDMOVING vs FIX-MOVING). The resulting vector was convolved with the canonical HRF and was used as PPI regressor in the psychophysiological analyses on the first level (Friston et al., 1997). The two main effects of this interaction, i.e., the convolved difference between
ATTEND-MOVING and FIX-MOVING and the time course of the cerebellum, served as covariates in the analyses, which also included the six realignment parameters and an intercept. These first level models were estimated using the same methods for whitening the data as described above (temporal filtering and adjustment of autocorrelations). Parameter estimates of the PPI regressors were entered into a one-sample $t$ test at the group level and results were thresholded at $p<0.05$ (corrected at the cluster level, based on Gaussian random field theory, using a cluster-defining threshold of $p<0.001$, uncorrected).

The PPI analyses were used to identify cortical regions for subsequent modeling that showed psychological (attentional) effects on linear estimates of coupling - in terms of attention-dependent changes in the influence of remote (cerebellar) influences. The effective connectivity among three of these cortical regions within the dorsal visual stream and the cerebellar region crus I was then examined with DCM to characterize the modulatory effects of attention within this network.

Dynamic causal modeling and Bayesian model selection. We applied DCM to characterize dynamic changes in effective connectivity of four regions or nodes, identified by the previous PPI analysis, namely the right cerebellum (CBL), primary visual cortex (V1), left secondary visual cortex (V5), and left posterior parietal cortex (PPC). These were selected primarily because the PPI results showed enhanced connectivity with the cerebellum (see below). Indeed, effective connectivity has previously been analyzed for these same regions except the cerebellum (Friston and Büchel, 2000).

In brief, DCM uses an explicit forward model which tries to explain how a measured signal is caused at the neuronal level (Friston et al., 2003). For fMRI, this forward model is based on the balloon model (Buxton et al., 1998). The dynamics of the system are described using a bilinear state equation that includes context-independent, average (or endogenous) connectivities between the nodes (conventionally referred to as matrix A) and two context-dependent terms: the modulatory input matrix B and the driving input $\mathrm{C}$ (see Eq. 1). The modulatory input acts dynamically on the strengths of the average connectivities and is realized for example by experimentally changing the context (e.g., by instruction to shift attention). The driving inputs 
refer to direct or exogenous inputs by which the system is perturbed directly at one or more nodes (e.g., by sensory stimulation).

$$
\frac{d x}{d t}=\left(A+\sum_{i=1}^{m} u_{i} B^{(i)}\right) x+C u .
$$

DCM analyses were performed using DCM10 of the SPM8 software package and were processed at the high performance computing cluster of the Center for Computing and Communication of RWTH Aachen University. It should be emphasized that the present study focuses on inference on model structure and not on model parameters. For this purpose, Bayesian model selection was used to identify the most probable model given the data among the models defined in the model spaces (Stephan et al., 2009). Although attention is considered to be a higherorder cognitive function, we assumed that the physiological mechanisms underlying the task in the present study do not vary much across subjects. In other words, we assumed that all subjects in our sample would "prefer" the same model, so that the inference method for model selection was based on fixed effects. We used a greedy search strategy to optimize the dynamic causal models. First, we identified where attention enters the network as an exogenous influence on regional activity (allowing for attentional modulation of extrinsic and intrinsic connections). Having identified the most likely source of attention-related responses, we then searched for the best combination of extrinsic (between regions) connections that showed attentional modulation.

\section{Results}

\section{Attention-related activity and PPI analysis}

Our whole-brain statistical parametric mapping identified several key regions showing main effects of stimulus and attention. In brief, these included the inferior frontal gyri, the inferior parietal lobules and the supramarginal gyri bilaterally as well as superior medial gyrus and middle frontal gyrus of the left hemisphere. Activation clusters in the right hemisphere were observed in superior and middle orbital gyri and in crus I of the cerebellum (see Fig. 2 and Table 1 for details).

The PPI analyses revealed attention-related modulations of cerebellar inputs to a large part of the dorsal visual stream, including primary and secondary visual cortices (V1 to V5). Beyond that, the functional network included posterior parietal cortex and it was quite conspicuous that the connectivity of the cerebellum during attention was largely confined to these visual areas (apart from a cluster in left prefrontal cortex; Fig. 3, Table 2).

\section{Definition of model spaces and posteriors of DCM analyses}

Clearly, the number of models that accommodate attentional modulations in our extended network is large. Therefore, we used a heuristic (greedy) search of models with the following two steps. First, we identified the most plausible region or regions responding directly to attention, using an exhaustive model search over all combinations of direct attentional effects and modulatory attentional effects limited to connections with the cerebellum. Having established where attention has direct effects, we then searched a new model space that allowed for attentional modulation of all connections.

In more detail, all models tested in this study had a full endogenous connectivity structure among the four nodes, i.e., there were 12 connections between nodes (not including self connections). Furthermore, all models were perturbed by any visual input at V1 only, whereas any moving visual input (attended or not attended) modulated the connectivity from V1 to V5. As a first step, we tested where in the system attention perturbs the system as a driving input. With four nodes, there are $2^{4}=16$
Table 1. Activation clusters related to attention to moving objects

\begin{tabular}{|c|c|c|c|c|c|c|}
\hline \multirow[b]{2}{*}{ Anatomical label } & \multirow{2}{*}{$\begin{array}{l}\text { Anatomy } \\
\text { toolbox }\end{array}$} & \multirow{2}{*}{$\begin{array}{l}\text { Cluster } \\
\text { size }\end{array}$} & \multirow[b]{2}{*}{ Z-score } & \multicolumn{3}{|c|}{ MNI coordinates } \\
\hline & & & & $x$ & $y$ & $z$ \\
\hline R supramarginal gyrus & $\mathrm{IPC}(\mathrm{PF})$ & 308 & 6.3753 & 49.5 & -39 & 42 \\
\hline \multirow{2}{*}{$\begin{array}{l}\text { R inferior frontal gyrus, pars } \\
\text { orbitalis }\end{array}$} & \multirow[t]{2}{*}{ Insula } & \multirow[t]{2}{*}{435} & 6.0836 & 34.5 & 25.5 & -9 \\
\hline & & & 5.6522 & 42 & 24 & -3 \\
\hline L inferior parietal lobule & $\mathrm{IPC}(\mathrm{PF})$ & 98 & 5.9156 & -46.5 & -46.5 & 42 \\
\hline $\begin{array}{l}\text { L inferior frontal gyrus, pars } \\
\text { triangularis }\end{array}$ & BA 45 & 116 & 5.7067 & -51 & 18 & 0 \\
\hline R superior orbital gyrus & & 70 & 5.6957 & 22.5 & 49.5 & -16.5 \\
\hline \multirow[t]{3}{*}{ L superior medial gyrus } & & 399 & 5.5315 & 0 & 28.5 & 37.5 \\
\hline & & & 5.5191 & -7.5 & 22.5 & 39 \\
\hline & & & 5.3673 & 3 & 37.5 & 34.5 \\
\hline \multirow[t]{2}{*}{ R inferior parietal lobule } & hlP1 & 70 & 5.4628 & 42 & -51 & 43.5 \\
\hline & IPC (PGa) & & 4.9753 & 42 & -52.5 & 52.5 \\
\hline $\mathrm{L}$ middle fro & BA 45 & 24 & 5.3970 & -51 & 27 & 31.5 \\
\hline \multirow[t]{3}{*}{ R cerebellum } & \multirow[t]{3}{*}{ lobule VIla crus I } & 159 & 5.3937 & 42 & -55.5 & -37.5 \\
\hline & & & 5.2304 & 39 & -61.5 & -33 \\
\hline & & & 5.1037 & 28.5 & -67.5 & -30 \\
\hline L middle fron & & 25 & 5.3801 & -33 & 51 & 0 \\
\hline \multirow{3}{*}{$\begin{array}{l}\text { Rinferior frontal gyrus, pars } \\
\text { opercularis }\end{array}$} & BA 45 & 291 & 5.3671 & 51 & 18 & 37.5 \\
\hline & BA 44/45 & & 5.3484 & 57 & 18 & 28.5 \\
\hline & BA 44 & & 5.1530 & 45 & 12 & 31.5 \\
\hline \multirow{3}{*}{$\begin{array}{l}\text { L inferior frontal gyrus, pars } \\
\text { triangularis }\end{array}$} & BA 45 & 16 & 5.2272 & -37.5 & 25.5 & 27 \\
\hline & BA 44/45 & 21 & 5.1695 & -42 & 22.5 & 9 \\
\hline & BA 44/45 & 7 & 5.0527 & -43.5 & 27 & 25.5 \\
\hline L supramarginal gyrus & \multirow[t]{2}{*}{$\mathrm{IPC}(\mathrm{PF})$} & 8 & 4.9995 & -63 & -46.5 & 24 \\
\hline R middle orbital gyrus & & 5 & 4.9838 & 30 & 55.5 & -9 \\
\hline (L) supplementary motor area & BA 6 & 11 & 4.9626 & 0 & 12 & 55.5 \\
\hline $\begin{array}{l}\text { R inferior frontal gyrus, pars } \\
\text { opercularis }\end{array}$ & BA 45 & 1 & 4.9393 & 48 & 18 & 25.5 \\
\hline $\begin{array}{l}\text { L inferior frontal gyrus, pars } \\
\text { triangularis }\end{array}$ & BA 44 & 1 & 4.9258 & -45 & 21 & 3 \\
\hline L precentral gyrus & BA 6 & 1 & 4.9121 & -45 & 4.5 & 49.5 \\
\hline
\end{tabular}

Regions with enhanced activity in the ATTEND-MOVING condition versus the FIX-MOVING condition, thresholded at $p<0.05$ corrected for multiple comparisons at the voxel level. The second column (Anatomy toolbox) gives labels described in more detail by Eickhoff et al. (2007). R, Right; L, left.

possible combinations of input regions where this may happen. This includes the possibility that attention does not enter the system at one of the nodes, but rather just modulates connections between nodes. For each of these 16 combinations, we defined 64 models that differed regarding the existence of modulatory inputs of attention on the connections between the cerebellum and one or more of the other three nodes. As there are six possible connectivities between the cerebellum and three other regions (three from and three to the cerebellum), the specified model space was exhaustive $\left(2^{6}=64\right)$ with respect to these modulatory inputs of attention. For all other modulatory inputs, we temporarily assumed that attention affects all other six connections between V1, V5, and PPC in an equivalent way. Accordingly, the model space consisted of $16 \times 64=1024$ models where the space was partitioned into the 16 families of models that differed with respect to the driving input of attention. Inference was made at the family level (Penny et al., 2010) and the results clearly suggest that attention has a single driving input on PPC with a posterior probability of $99.94 \%$.

After concluding that attention has most likely direct effects only on PPC we proceeded with a second step for which a new model space was defined. This model space addressed the question which connections between nodes were modulated by attention. With 12 connections being potentially modulated there are $2^{12}=4096$ possible combinations of attention-modulated connections. Thus, we built an exhaustive model space in this respect consisting of all 4096 models, all of which possessed a driving input of attention on PPC and shared all properties described 


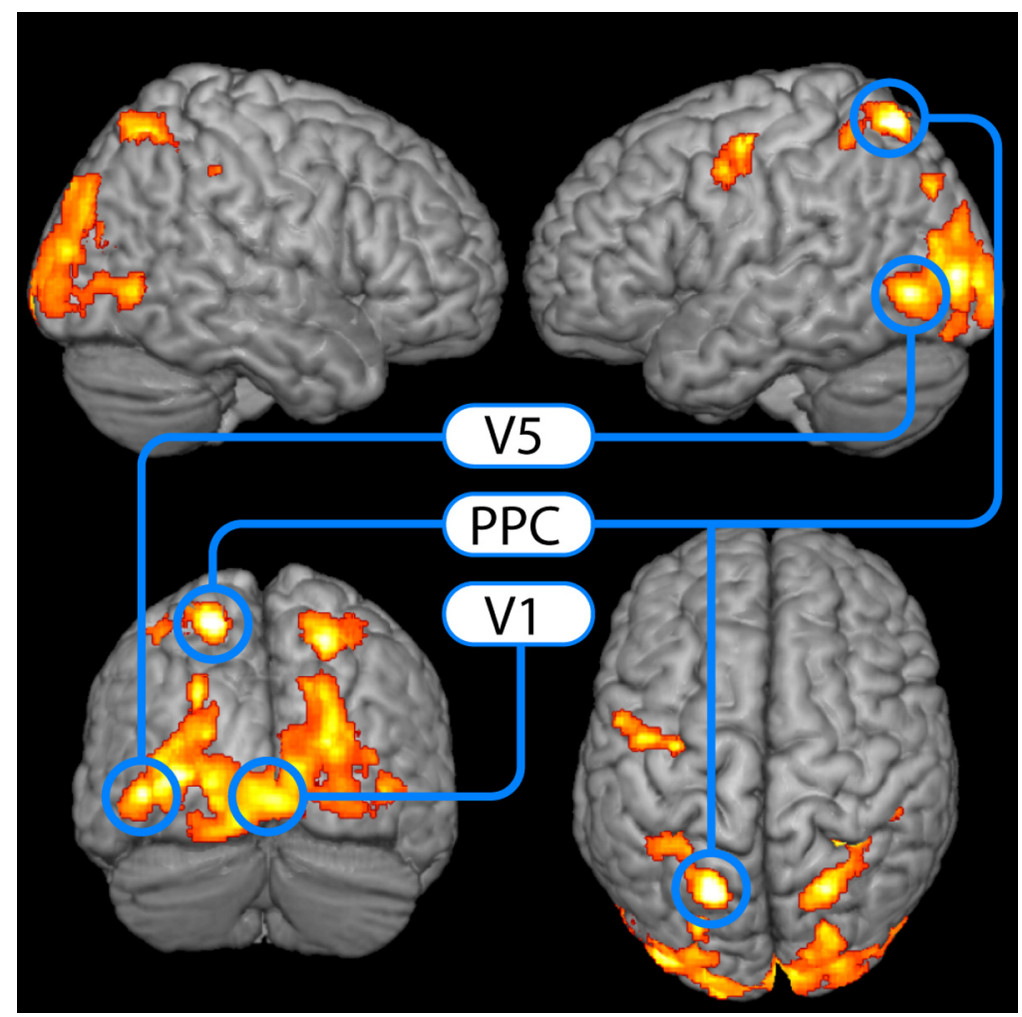

Figure 3. Results for the psychophysiological interaction between attention and the cerebellum rendered on an anatomical brain image. The suprathreshold regions show a psychophysiological interaction with the cerebellum during attention compared with mere watching moving stimuli ( $p<0.05$ corrected at the cluster level using $p<0.001$ uncorrected for cluster definition). Regions marked in blue (in addition to right crus I) were selected for subsequent DCM analyses. Upper left, View from right; upper right, view from left; lower left, view from behind; lower right, view from top.

Table 2. Results of the psychophysiological interaction with the cerebellum as seed region

\begin{tabular}{lllllll}
\hline & \multirow{2}{*}{$\begin{array}{l}\text { Anatomy } \\
\text { Anatomical label }\end{array}$} & \multirow{2}{*}{$\begin{array}{l}\text { Cluster } \\
\text { toolbox }\end{array}$} & & \multicolumn{4}{c}{ MNI coordinates } \\
\cline { 5 - 7 } & size & Z-score & $x$ & $y$ & z \\
\hline L superior parietal lobule & SPL (7a) & 979 & 5.4776 & -19.5 & -64.5 & 61.5 \\
L inferior parietal lobule & SPL (7a) & & 3.9466 & -36 & -48 & 54 \\
& BA 2 & & 3.4293 & -27 & -45 & 49.5 \\
L middle occipital gyrus & hOC3v (V3v) & 11746 & 4.9732 & -33 & -88.5 & 3 \\
L inferior occipital gyrus & hOC5 (V5) & & 4.9487 & -45 & -70.5 & -3 \\
R superior occipital gyrus & & & 4.7993 & 22.5 & -82.5 & 16.5 \\
R superior parietal lobule & SPL (7a) & 1509 & 4.8866 & 22.5 & -63 & 55.5 \\
& SPL (7PC) & & 4.8287 & 28.5 & -48 & 46.5 \\
R inferior parietal lobule & hIP3/SPL (7PC) & & 4.0255 & 36 & -45 & 45 \\
L middle occipital gyrus & SPL (7a) & 259 & 4.5774 & -25.5 & -76.5 & 34.5 \\
L precentral gyrus & BA 6 & 509 & 4.5263 & -33 & -10.5 & 54 \\
& & & 4.4678 & -46.5 & -7.5 & 54 \\
& & & 4.1659 & -54 & -1.5 & 43.5 \\
\hline
\end{tabular}

The specified regions exhibit a psychophysiological interaction with the seed region (right cerebellum) during attention to moving stimuli compared with watching moving stimuli (ATTEND-MOVING vs FIX-MOVING). Results were thresholded at $p<0.05$ corrected for multiple comparisons at the cluster level using a cluster-forming threshold of $p<0.001$ at the voxel level. The second column (Anatomy toolbox) gives labels described in more detail by Eickhoff et al. (2007). R, Right; L, left.

above (full endogenous connectivity, driving input of visual stimuli on V1, modulatory input of moving visual stimuli on the $\mathrm{V} 1 \rightarrow \mathrm{V} 5$ connection). Contrary to our expectation, the posteriors sharply peaked on one model (Fig. 4) which had a posterior probability of $75.09 \%$ (model 382). The odds ratio of this posterior and that of the second best model $\left(\operatorname{model} 1528, p\left(m_{1528} \mid Y\right)=\right.$ 0.0625 ) was 12.01 indicating positive to strong evidence in favor of the best compared with the second best model (Kass and Raf- tery, 1995). The structure of the winning model had two modulatory inputs of attention, namely on the connections from the cerebellum to V5 and from V5 to posterior parietal cortex (Fig. 5).

The parameters from the model in Figure 5 were tested for significance by twotailed one-sample $t$ tests, which were corrected for multiple comparisons (17 tested parameters). All driving inputs as well as the modulatory input of moving stimuli on the V1 $\rightarrow \mathrm{V} 5$ connection were significantly larger than zero. Contrary to our expectations, the influence that V5 exerted on PPC significantly decreased under attention [mean $(\mathrm{M})=-0.694$ and $\mathrm{SE}= \pm 0.177]$. Conversely, the effective connectivity of the cerebellum on V5 increased during attention $(\mathrm{M}=0.902$ and $\mathrm{SE}= \pm 0.319)$. Parameters for the endogenous connections are summarized in Table 3 for all non-zero coupling parameters. Remarkably, the model shows a loop-like architecture for the endogenous connections including V5, PPC, and the cerebellum.

\section{Discussion}

In the PPI analysis, we demonstrated dynamic changes in effective connectivity of the cerebellum with other brain regions that were driven by increased visual attention. These regions showing this enhanced connectivity with the cerebellum where almost exclusively located in the dorsal visual stream (including V5 and PPC), which is associated with spatial localization of objects (PPC: Corradi-Dell'Acqua et al., 2008; Lane et al., 2011), motion perception (V5: Wilms et al., 2005; Orban, 2011) and spatial attention (PPC: Fan et al., 2005; Malhotra et al., 2009; Rawley and Constantinidis, 2010; Kellermann et al., 2011; Lane et al., 2012). Before we turn to the discussion of the consequential analyses of dynamic causal modeling, we briefly review some theoretical considerations regarding the functionality of the cerebellum.

\section{Theoretical Considerations about Cerebellar Functioning}

Converging theoretical foundations of cerebellar functioning have been formulated by several authors (Schmahmann, 1997; Wolpert et al., 1998) and have been augmented more recently (Paulin, 2005; Ito, 2008; Miall and King, 2008). Generally speaking, these theories act on the need of (even simple) organisms to interact with their environment, which is arranged of (moving) objects in space and time. To do that efficiently, dynamically changing states of the environment as well as of the organism itself have to be accounted for. To this end, cerebellar-like architecture evolved naturally to implement dynamic state estimators (or internal models) as so called "particle filters" using spiking neurons (Paulin, 2005). From our point of view, this conception might explain three properties of the cerebellum that have been associated with it: (1) By reason of its cytoarchitectonic uniformity, it is very likely that its computational or processing capabilities are likewise uniform (Ramnani, 2006). (2) Lesions in the cerebellum (at least in its hemispheres) are rarely accompanied 


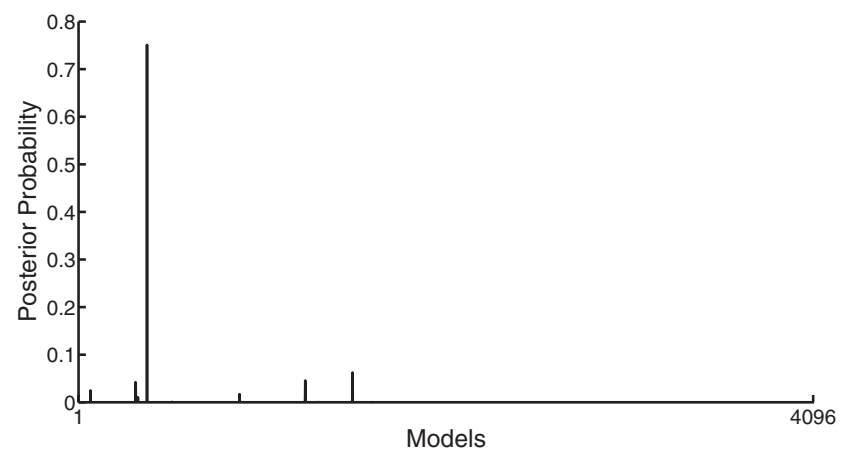

Figure 4. Distribution of the posterior probabilities among the model space for the 4096 bilinear models that differ with respect to the connection(s) that is/are modulated by attention. Posterior probabilities peak sharply on model 382 , which has a posterior of $75.09 \%$. The order of the models along the $x$-axis is arbitrarily chosen.

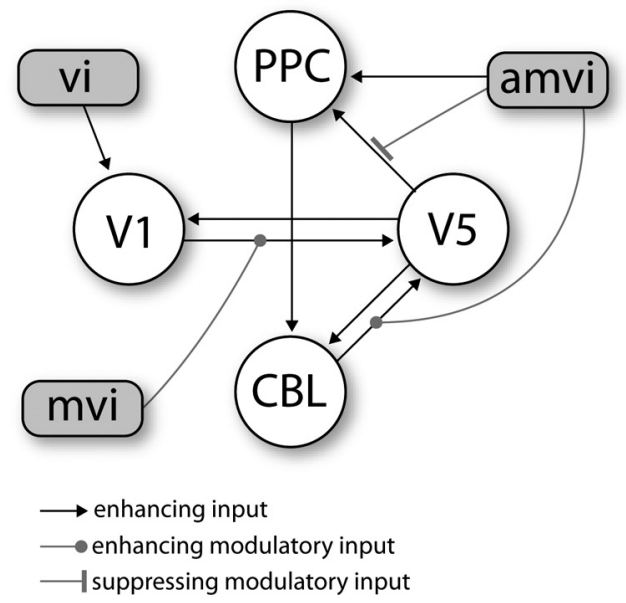

Figure 5. Depiction of the winning bilinear model according to the Bayesian model selection procedures. Shown are all connections and inputs whose parameters were significantly different from zero. vi, Visual input; mvi, moving visual input; amvi, attention to moving visual input.

Table 3. Endogenous connectivities for the winning model (depicted in Figure 5)

\begin{tabular}{lllll}
\hline To/From & V1 & \multicolumn{1}{l}{ V5 } & PPC & CBL \\
\hline V1 & - & $0.698( \pm 0.108)$ & 0 & 0 \\
V5 & 0 & - & 0 & $-0.284( \pm 0.084)$ \\
PPC & 0 & $0.444( \pm 0.044)$ & - & 0 \\
CBL & 0 & $-0.205( \pm 0.042)$ & $0.499( \pm 0.049)$ & -
\end{tabular}

Significant parameters are listed for task-independent and therefore endogenous connectivities between the four selected regions of the DCM analyses. Parameters were selected according to their significant non-zero values with their means and SEs in parentheses. Significance was assessed via corrected one-sample $t$ test for 17 two-sided comparisons for each model. Azero denotes nonsignificant parameters, whereas the dashes for the self-connections indicate that these parameters were not tested. Parameters are shown for the winning model, which is depicted in Figure 5 .

by specific and/or complete losses of functionality for which reason it has been labeled as "useful but not necessary" (Bower, 1997). It should be noted that from an evolutionary perspective with its selection pressure a gain in speed, accuracy and smoothness of motor responses and sensory data acquisition might very well be necessary to escape predators or to pursue prey successfully. (3) There are about as many neurons in the cerebellum as there are in the cerebrum and an increase in size of the cerebrum comes along with an according increase of the cerebellum during phylogeny. This simultaneous growth of the two structures can well be reconciled with the computational uniformity of the cerebellum and the distinct functional topography of corticocer- ebellar loops (Habas et al., 2009; Krienen and Buckner, 2009; O’Reilly et al., 2010; Buckner et al., 2011).

\section{Effective connectivity of the cerebellum}

We used dynamic causal modeling and Bayesian model selection to infer the most probable model structure among the cerebellum, V1, V5, and PPC. This method affords conclusions about several properties of the system and its responses to external input: (1) specification of driving and modulatory inputs (effects of external inputs on nodes and on connections between nodes, respectively) and (2) specification of the influence that one node exerts over another. The winning model manifests a cerebrocerebellar loop with endogenous, reciprocal projections between V5 and the cerebellum, and another endogenous or average connection from PPC to the cerebellum and from V5 to PPC (Table 3). Note that there are no monosynaptic connections between the cerebrum and the cerebellum (Buckner et al., 2011): efferent projections from the cerebrum are mediated via pontine nuclei (pontocerebellar tract) and afferents are connected by way of deep cerebellar nuclei and the thalamus (dentothalamocortical tract).

Under attention the $\mathrm{CBL} \rightarrow \mathrm{V} 5$ connection is enhanced, whereas the V5 $\rightarrow$ PPC connection is suppressed (Fig. 5), which seems to be counterintuitive at first glance. Keeping in mind that visual motion was highly predictable in the present study our results are consistent with a modulatory role of attention in predictive coding formulations of V5 motion processing (Feldman and Friston, 2010). In predictive coding, attention is modeled by an increase in the precision or gain of cells encoding prediction error, when there is less uncertainty about that prediction error. From the point of view of predictive coding, the bilinear model selected makes perfect sense: attention (presumably mediated by prefrontal or parietal sources) increases the sensitivity of populations in V5 to cerebellar inputs, while decreasing the sensitivity of higher-level (PPC) populations to inputs from V5. This means that there is a selective enhancement of the precision of motion processing responses when attending to motion. Crucially, this increased sensitivity of V5 appears to be selective for cerebellar inputs. This may reflect the role of the cerebellum as a state estimator providing (top-down) predictions about motion in the attentive conditions. The relative suppression of PPC sensitivity to bottom up information is again entirely consistent with a hierarchical redeployment of precision or gain in hierarchical Bayesian inference, on which predictive coding rests.

\section{State estimations of the cerebellum during visual attention}

Against this background, we speculate that the cerebellum performs uniform computations on highly specific information, which it receives from corresponding "specialized" areas of the cerebral cortex. Consequently, neither the degree of specialization nor the hierarchical level of (cognitive) processing is determined by the cerebellum. Instead the cerebrocortical areas from which the cerebellum receives inputs determine the degree of specialization. Similarly, the directionality of processing (bottom-up or top-down) depends on the hierarchical position of the cortical area from which the cerebellum receives its inputs relative to the cortical area to which the cerebellum directs its outputs. In the selected model the proposed state estimations performed by the cerebellum are not sent back to the cerebral origin of the information. Instead, the cerebellar output acts on a spatially distant and functionally different cortical area (compared with the origin), which is also on a lower hierarchical level of processing. As outlined above, this cerebrocerebellar loop is 
consistent with predictive coding formulations of attention to highly predictable stimuli, where the sensitivity of lower levels (V5) to inputs from higher levels (PPC via cerebellum) is increased. This interpretation implies that state estimates, which are computed by crus I of the cerebellum-based on information provided by the PPC - are fed to V5. Crucially, the sensitivity of V5 populations to these cerebellar inputs is increased during attention (presumably through modulatory effects of parietal and frontal afferents) to provide precise top-down predictions about moving and therefore predictable stimuli.

The most efficient way to detect (putative) changes in velocity of constantly moving stimuli would be to instantiate an internal model about the expected velocity and align these internal models with actual inputs. As long as the predictions stored in the loop are successfully aligned to external inputs there is no need to interrupt and update the internal model, albeit this possibility is given in the model by strong signals from V5. According to these considerations, the cerebellum would be of great importance to support the detection of prediction errors and thereby supporting predictive coding (Rao and Ballard, 1999; Langner et al., 2012; Schlerf et al., 2012). On a perceptual level, crus I has previously been associated with the cancellation of eye-movement-induced retinal image motion (Lindner et al., 2006), which might support the possible role of the cerebellum in predictive coding during visual processing.

There are several important aspects, however, that have not been addressed in this study. For example, we expected the cerebellum to also interact with prefrontal regions, which was not the case with respect to the PPI analysis. Nevertheless, the prefrontal cortex was engaged during attention which brings forth questions about putative changes within the network structure when these regions were also considered in the models. Even more important is the question how the system dynamics respond to actual changes in velocity of the stimuli when the participant detects them. The putative role of the cerebellum in predictive coding has to be addressed in experiments where behavioral data along with a classification of answer correctness are available.

\section{Conclusions}

The present study provides empirical evidence for theories of cerebellar functioning which assume that the cerebellum facilitates predictions of dynamic perceptual events. We identified a cerebrocerebellar loop consisting of posterior parietal cortex, V5 and the cerebellum, the latter supposedly maintaining internal models about visual inputs. In cases of highly predictable visual input the sensitivity of posterior parietal populations to bottom-up V5 efferents is attenuated, while the influences of cerebellar afferents to the V5 region are enhanced. In other words, attentional mechanisms appear to emphasize parietocerebellar influences on motion processing in V5 through a selective disengagement of bottom-up V5 inputs to parietal cortex and an augmentation of top-down parietocerebellar influences. This imposes at least two questions for future studies: (1) How does the predictability of perceptual events or the manipulation of velocity act on the system? (2) How do other regions or even other cerebrocerebellar loops fit into this network, especially medial prefrontal cortex and temporoparietal junction? Both questions need to be addressed to further elaborate on the role of the cerebellum in predictive coding formulations of attention.

\section{Notes}

Supplemental material for this article is available at http://www. ukaachen.de/go/show? $\mathrm{ID}=25009127 \& \mathrm{DV}=0 \& \mathrm{COMP}=$ download $\&$
NAVID $=4760197 \&$ NAVDV $=0$. Description of the methods for the analysis of the EEG data, which were simultaneously acquired with functional magnetic resonance imaging. This material has not been peer reviewed.

\section{References}

Ashburner J, Friston KJ (2005) Unified segmentation. Neuroimage 26:839-851.

Blackwood N, Ffytche D, Simmons A, Bentall R, Murray R, Howard R (2004) The cerebellum and decision making under uncertainty. Brain Res Cogn Brain Res 20:46-53.

Booth JR, Wood L, Lu D, Houk JC, Bitan T (2007) The role of the basal ganglia and cerebellum in language processing. Brain Res 1133:136-144.

Bower JM (1997) Control of sensory data acquisition. Int Rev Neurobiol 41:489-513.

Büchel C, Josephs O, Rees G, Turner R, Frith CD, Friston KJ (1998) The functional anatomy of attention to visual motion. A functional MRI study. Brain 121:1281-1294.

Buckner RL, Krienen FM, Castellanos A, Diaz JC, Yeo BTT (2011) The organization of the human cerebellum estimated by intrinsic functional connectivity. J Neurophysiol 106:2322-2345.

Buxton RB, Wong EC, Frank LR (1998) Dynamics of blood flow and oxygenation changes during brain activation: the Balloon model. Magn Reson Med 39:855-864.

Chen SH, Desmond JE (2005) Cerebrocerebellar networks during articulatory rehearsal and verbal working memory task. Neuroimage 24:332-338.

Corradi-Dell'Acqua C, Hesse MD, Rumiati RI, Fink GR (2008) Where is a nose with respect to a foot? The left posterior parietal cortex processes spatial relationships among body parts. Cereb Cortex 18:2879-2890.

Diedrichsen J (2006) A spatially unbiased atlas template of the human cerebellum. Neuroimage 33:127-138.

Eickhoff SB, Paus T, Caspers S, Grosbras MH, Evans AC, Zilles K, Amunts K (2007) Assignment of functional activations to probabilistic cytoarchitectonic areas revisited. Neuroimage 36:511-521.

Fan J, McCandliss BD, Fossella J, Flombaum JI, Posner MI (2005) The activation of attentional networks. Neuroimage 26:471-479.

Feldman H, Friston KJ (2010) Attention, uncertainty and free-energy. Front Hum Neurosci 4:215.

Frings M, Dimitrova A, Schorn CF, Elles HG, Hein-Kropp C, Gizewski ER, Diener HC, Timmann D (2006) Cerebellar involvement in verb generation: an fMRI study. Neurosci Lett 409:19-23.

Friston KJ, Büchel C (2000) Attentional modulation of effective connectivity from V2 to V5/MT in humans. Proc Natl Acad Sci U S A 97:7591-7596.

Friston KJ, Buechel C, Fink GR, Morris J, Rolls E, Dolan RJ (1997) Psychophysiological and modulatory interactions in neuroimaging. Neuroimage 6:218-229.

Friston KJ, Harrison L, Penny W (2003) Dynamic causal modelling. Neuroimage 19:1273-1302.

Graydon FX, Friston KJ, Thomas CG, Brooks VB, Menon RS (2005) Learning-related fMRI activation associated with a rotational visuomotor transformation. Brain Res Cogn Brain Res 22:373-383.

Habas C, Kamdar N, Nguyen D, Prater K, Beckmann CF, Menon V, Greicius MD (2009) Distinct cerebellar contributions to intrinsic connectivity networks. J Neurosci 29:8586-8594.

Ito M (2008) Control of mental activities by internal models in the cerebellum. Nat Rev Neurosci 9:304-313.

Ivry R (1997) Cerebellar timing systems. Int Rev Neurobiol 41:555-573.

Kass RE, Raftery AE (1995) Bayes factors. J Am Stat Assoc 90:773-795.

Kellermann T, Reske M, Jansen A, Satrapi P, Shah NJ, Schneider F, Habel U (2011) Latencies of BOLD response during visual attention processes. Brain Res 1386:127-138.

Krienen FM, Buckner RL (2009) Segregated fronto-cerebellar circuits revealed by intrinsic functional connectivity. Cereb Cortex 19:2485-2497.

Lane AR, Smith DT, Schenk T, Ellison A (2011) The involvement of posterior parietal cortex in feature and conjunction visuomotor search. J Cogn Neurosci 23:1964-1972.

Lane AR, Smith DT, Schenk T, Ellison A (2012) The involvement of posterior parietal cortex and frontal eye fields in spatially primed visual search. Brain Stimul 5:11-17.

Langner R, Kellermann T, Eickhoff SB, Boers F, Chatterjee A, Willmes K, 
Sturm W (2012) Staying responsive to the world: modality-specific and -nonspecific contributions to speeded auditory, tactile, and visual stimulus detection. Hum Brain Mapp 33:398-418.

Lindner A, Haarmeier T, Erb M, Grodd W, Thier P (2006) Cerebrocerebellar circuits for the cancellation of eye-movement-induced retinal image motion. J Cogn Neurosci 18:1899-1912.

Malhotra P, Coulthard EJ, Husain M (2009) Role of right posterior parietal cortex in maintaining attention to spatial locations over time. Brain 132:645-660.

Miall RC, King D (2008) State estimation in the cerebellum. Cerebellum 7:572-576.

Orban GA (2011) The extraction of 3D shape in the visual system of human and nonhuman primates. Annu Rev Neurosci 34:361-388.

O'Reilly JX, Mesulam MM, Nobre AC (2008) The cerebellum predicts the timing of perceptual events. J Neurosci 28:2252-2260.

O'Reilly JX, Beckmann CF, Tomassini V, Ramnani N, Johansen-Berg H (2010) Distinct and overlapping functional zones in the cerebellum defined by resting state functional connectivity. Cereb Cortex 20:953-965.

Paulin MG (1997) Neural representations of moving systems. Int Rev Neurobiol 41:515-533.

Paulin MG (2005) Evolution of the cerebellum as a neuronal machine for Bayesian state estimation. J Neural Eng 2:S219-S234.

Penny WD, Stephan KE, Daunizeau J, Rosa MJ, Friston KJ, Schofield TM, Leff AP (2010) Comparing families of dynamic causal models. PLoS Comput Biol 6:e1000709.

Ramnani N (2006) The primate cortico-cerebellar system: anatomy and function. Nat Rev Neurosci 7:511-522.

Rao RP, Ballard DH (1999) Predictive coding in the visual cortex: a func- tional interpretation of some extra-classical receptive-field effects. Nat Neurosci 2:79-87.

Rawley JB, Constantinidis C (2010) Effects of task and coordinate frame of attention in area $7 \mathrm{a}$ of the primate posterior parietal cortex. J Vis 10:1201-1216.

Salmi J, Pallesen KJ, Neuvonen T, Brattico E, Korvenoja A, Salonen O, Carlson S (2010) Cognitive and motor loops of the human cerebrocerebellar system. J Cogn Neurosci 22:2663-2676.

Schlerf J, Ivry JB, Diedrichsen J (2012) Encoding of sensory prediction errors in the human cerebellum. J Neurosci 32:4912-4922.

Schmahmann JD (1991) An emerging concept: The cerebellar contribution to higher function. Arch Neurol 48:1178-1187.

Schmahmann JD (1997) The cerebellum and cognition. San Diego: Academic.

Schmahmann JD, Sherman JC (1998) The cerebellar cognitive affective syndrome. Brain 121:561-579.

Sokolov AA, Erb M, Gharabaghi A, Grodd W, Tatagiba MS, Pavlova MA (2012) Biological motion processing: the left cerebellum communicates with right superior temporal sulcus. Neuroimage 59:2824-2830.

Stephan KE, Penny WD, Daunizeau J, Moran RJ, Friston KJ (2009) Bayesian model selection for group studies. Neuroimage 46:1004-1017.

Stoodley CJ, Schmahmann JD (2009) Functional topography in the human cerebellum: a meta-analysis of neuroimaging studies. Neuroimage 44:489-501.

Wilms M, Eickhoff SB, Specht K, Amunts K, Shah NJ, Malikovic A, Fink GR (2005) Human V5/MT+: comparison of functional and cytoarchitectonic data. Anat Embryol 210:485-495.

Wolpert DM, Miall RC, Kawato M (1998) Internal models in the cerebellum. Trends Cogn Sci 2:338-347. 